\title{
Developing a General Methodology for Driving Cycle Construction: Comparison of Various Established Driving Cycles in the World to Propose a General Approach
}

\author{
Uditha Galgamuwa, Loshaka Perera, Saman Bandara \\ Transportation Engineering Division, Department of Civil Engineering, University of Moratuwa, Moratuwa, \\ Sri Lanka \\ Email: nandun8781@gmail.com
}

Received 1 July 2015; accepted 27 September 2015; published 30 September 2015

Copyright (C) 2015 by authors and Scientific Research Publishing Inc.

This work is licensed under the Creative Commons Attribution International License (CC BY).

http://creativecommons.org/licenses/by/4.0/

c) (i) Open Access

\begin{abstract}
Many models have been developed in the world to estimate emission inventories and fuel consumption in the past and those models can be broadly categorized as either a travel based model or a fuel based model. Driving cycles can be considered as one of the major travel based models to estimate emission inventories. It can be used for various purposes such as setting up the emission standards, for traffic management purposes and also to determine the travel time. In the past, researchers have tried to use readily available, well established driving cycles in their environment which is different from the origin of the driving cycle in many aspects. Thus, the attempts have failed to give good quality results. This study attempts to critically evaluate the different methods used for driving cycle construction in different parts of the world under various conditions to propose a general suitable approach to develop a representative and economical driving cycle(s) for a given geographic location for set objectives.
\end{abstract}

\section{Keywords}

Driving Cycle, Driving Pattern, Driving Cycle Evaluation Parameters, Emission Estimation

\section{Introduction}

There are two major ways of estimating emission inventories and fuel consumption, namely, travel based models and fuel based models [1]. There are many fuel based models around the world which are used to estimate fuel consumption and emission inventories such as traffic flow models and traffic emission models [2], instan- 
taneous emission models [3] [4], average speed emission models [3] and using independent driving pattern factors [5]. In general, these models use top to bottom approach, where, the results were measured first and then try to identify the causes for the results. The methodology, driving cycle, falls under travel based models and it's been used to estimate fuel consumption and to develop emission inventories. The bottom-up approach is used to develop driving cycles, where prior to data collection, spatial and temporal characteristic of the routes or a region is identified. A driving pattern which influences exhaust emission and fuel consumption [6] can be illustrated based on such data and the prime advantage of this travel based method of driving cycle development is the less capital requirement compared to many other available methods.

Driving Cycle can be defined as a series of data points representing speed verses time [7], speed and gear selection as a function of time [8], speed versus distance [9] or time vs gradient [10], in a specific region or a part of a road segment. Driving Cycles are used for many purposes such as traffic engineering purpose [11] estimation of emission inventories [8] [12] and estimation of fuel consumption [13]. Figure 1 shows a sample driving cycle developed for traffic engineering purpose for Baseline road, Colombo, Sri Lanka while Figure 2 shows a sample driving cycle developed based on a case study to estimate emission inventories in Sri Lanka [14]. For the estimation of fuel consumption and emission inventories vehicles are tested using chassis dynamometers with respect to a given driving cycle [15]-[18].

Driving Cycle development was initiated in early 70's in some regions of the world and driving cycles can be broadly separated in to two segments, namely, "Transient Driving Cycles", which are developed using on road data and the "Modal" or "Polygonal" Cycles, which are developed by composing the collected data using a selected mechanism [7]. FTP 72 and FTP 75 can be considered as first two driving cycles that were developed in the world [19] and afterwards many improvements are being made to driving cycles to symbolize the actual on road driving pattern.

USA, Europe, Australia and Asia can be identified as the four main regions where many countries have been developing driving cycles during last thirty to forty years. Route selection, data collection, cycle construction [19] and cycle assessment [14] have been identified as four major common steps in driving cycle development. However, different countries have used various approaches in driving cycle development based on the time constrains, resource availability and information availability, and thus outputs have varied. Therefore, this paper tries to critically evaluate the methods used by different countries for each step with respect to different traffic behaviors and varied objectives. With this analysis a new developers would be able to find out the appropriateness of different methods that can be used for their driving cycle development at prevailing conditions or resources based on the purpose of the driving cycle development.

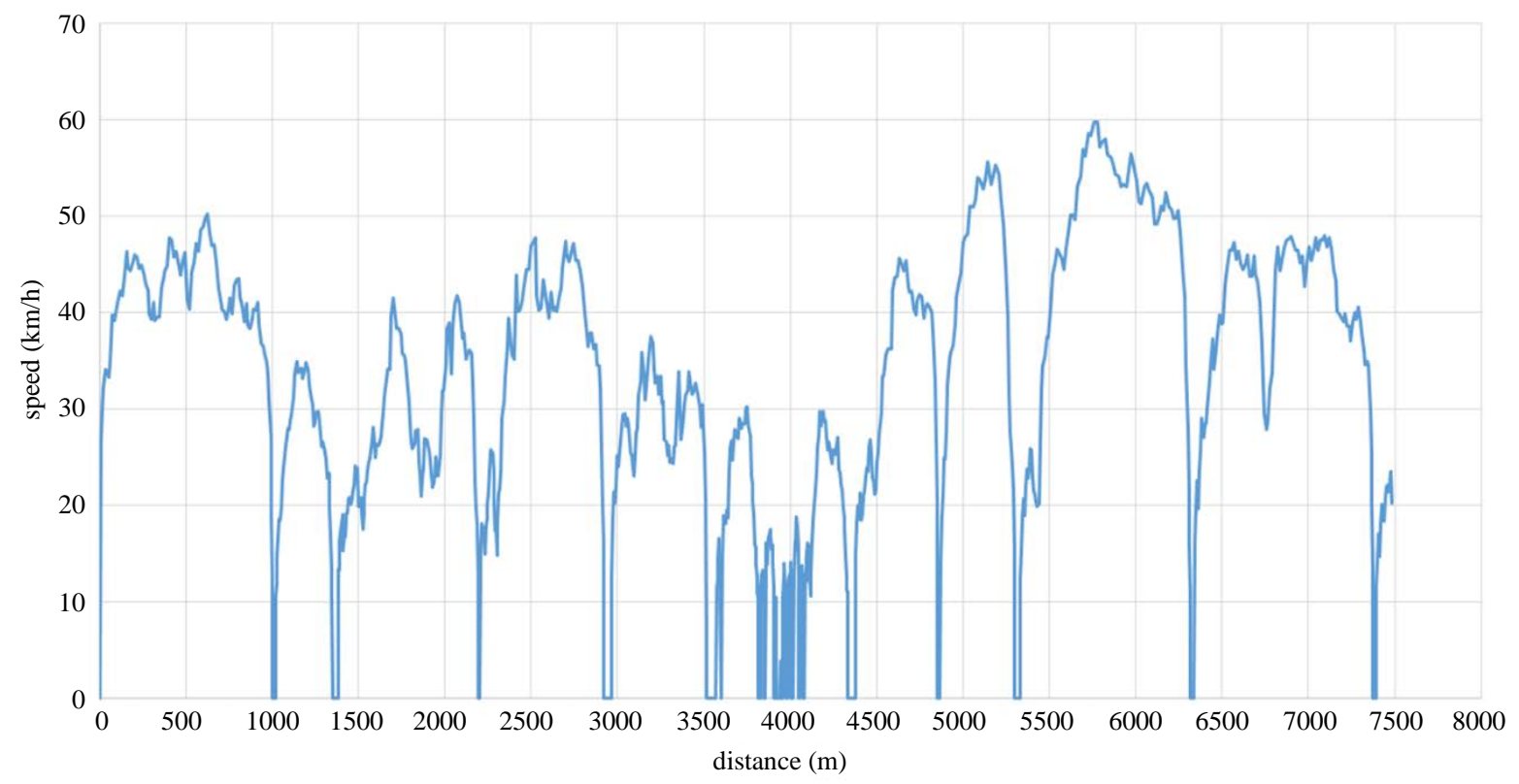

Figure 1. Driving cycle for traffic engineering purpose (sample). 


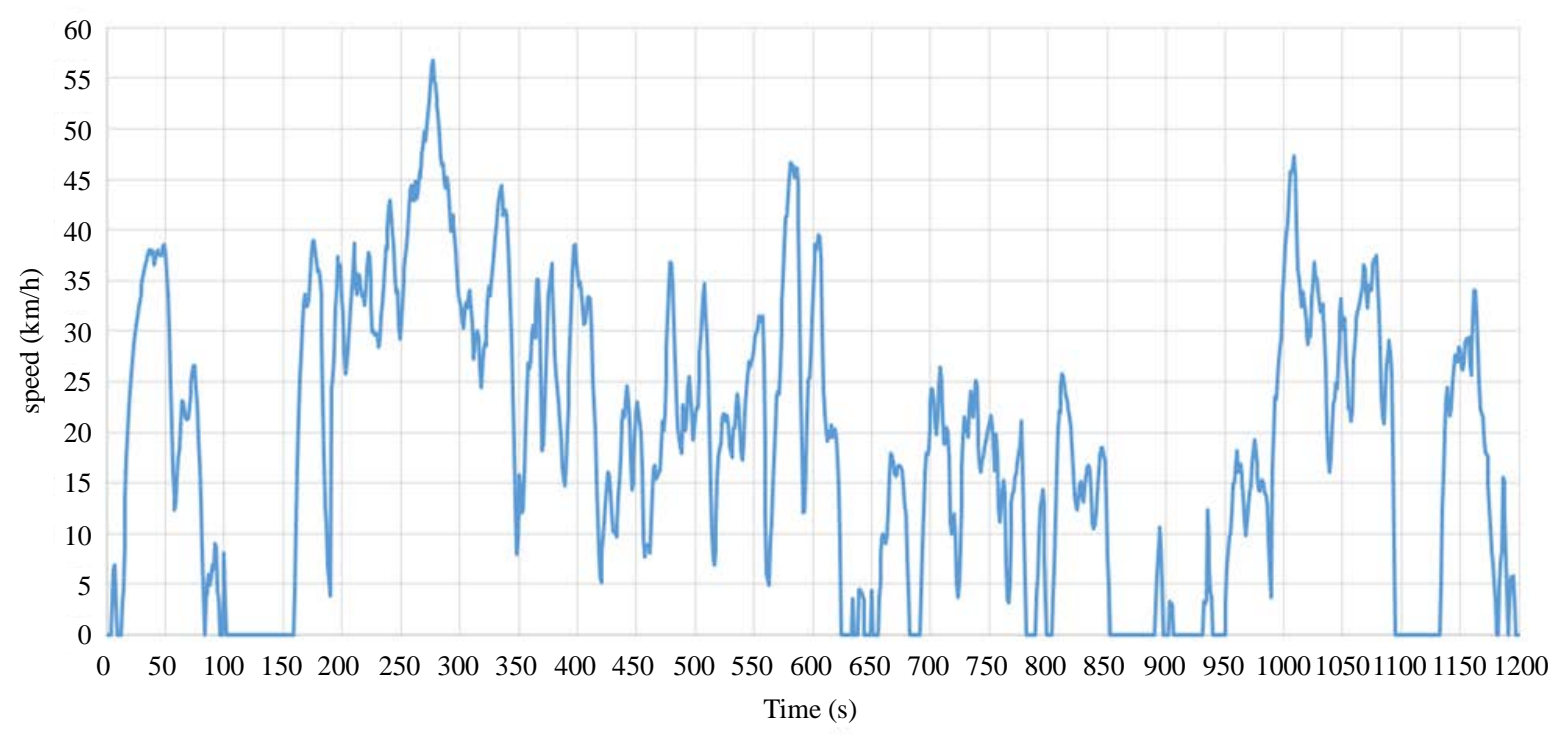

Figure 2. Driving cycle for emission purpose (sample).

\section{Comparison of Available Driving Cycles and Its Development Process}

\subsection{Route Selection}

Route selection is one of the major aspects in developing driving cycles. If routes selected are not representative of actual network to capture ordinary traffic behavior in that area, then the data collected will be biased, hence the results obtained from the driving cycle will indicate a deviation from its actual situation. The selected traveling routes should best represent the actual network and the typical traffic flow conditions affected by spatial or temporal conditions such as land use, road type, topography, availability of signalized intersections and population density in that area [6] [7] [20]. Furthermore when selecting routes for driving cycle development, either for emission purpose or to estimate fuel consumption, it is essential to consider the vertical alignment of the road as well. Because gradient of the road has an impact over vehicles fuel consumption and it varies significantly on the micro segment scale [21]. In addition, on regional basis it is necessary to consider the weather condition on each selected route, where routes could be selected from various regions or from countries sometimes like in European driving cycle and the driving pattern usually vary due to the prevailing weather conditions [22]. Micro texture of the routes [23], altitude and temperature are also contributory factors [24] to determine emission levels. Thorough knowledge about the routes is necessary in order to assign weightage for each factor to capture its significance. Therefore, driving cycle development is a subjective process fit into the purpose of driving cycle development or the researcher [7].

In Pune, India, five major roads (total length of $55 \mathrm{~km}$ ) which have higher Average Annual Daily Traffic (AADT) were selected as representative routes to collect data to develop a driving cycle [25]. A pre-defined set of origin/destination pairs were combined with highest overall AADT in Pune driving cycle and this was considered as a reference in determining the traffic density. Then the routes which are used frequently have been selected. In addition routes were selected based on time of the day which will represent the majority of vehicles according to AADT [19]. On the other hand, in Hong Kong (China), the route selection was mainly done based on the experience and knowledge of experts about local traffic conditions. Similarly for Colombo in Sri Lanka, route selection for a preliminary driving cycle development was mainly done based on the experience and local knowledge of professionals about the traffic behavior in Colombo area. In Colombo only one continuous road was selected to represent the area for data collection, mostly due to the prevailing financial constraint as well as lack of historical traffic data or resources. Relatively approach towards development of a preliminary driving cycle for Colombo has many limitations. Using researchers' experience to select routes can be biased and the researcher may not have considered all necessary parameters which needed to be considered such as origindestination patterns, mean trip length, vehicle kilometers, etc. Also literature suggest that the routes have to be weighted not according to the traffic volumes but according to the vehicle kilometers travelled on that road be- 
cause the traffic volumes represents the number of vehicle passing a particular point of the road where vehicle kilometers represent the total demand along the road segment. These will lead the selected sample biased and hence will over or under estimate the condition in the area. Nevertheless, with many draw backs this method requires lesser amount of data to be collected and easy selection of routes makes it more economical and thus better for countries developing their initial driving cycles. Further, this imprecise exercise enable developers or researchers to identify the necessary parameters to be considered for further developments. In a way it's better to identify the critical emission levels in a city if used major roads only.

The route selection of Bangkok driving cycle is based on the traffic flow data and the travel speed. Using traffic flow data, a traffic flow model was developed and hence travel speeds along each section of major roads are determined. Based on that the distribution of travel speeds of vehicles along the roads few major roads were selected which closely matched with all major roads previously considered. Selected roads are evaluated using some basic statistical parameters such as means and variance [26]. Advantage of this method is that it is easy to collect travel speeds along the road, hence the selection of routes is easy and economical. But the mean speed does not always represent the traffic condition of the road due to the fact that different vehicle mix may produce same mean speeds other road related parameters such as obstruction from the pedestrians and road surface conditions may also affect the mean speed.

In addition to Pune, Hong Kong (China) and Colombo driving cycles, route selection of Hanoi (Vietnam), driving cycle has considered travel activity patterns (OD travel patterns) and traffic flow characteristics. At initial stage there weren't any readily available historical traffic data. As a result, onsite observation, the knowledge and the experience of the researcher is heavily used when selecting routes emphasizing on four aspects, road type criteria (highways, main roads, collecting streets, and internal streets), route length criteria (according to the results from the survey the mean trip length was calculated), traffic volume criteria (most heavily congested roadways were identified) and land use criteria (according to the questionnaire survey) [27]. Even though it requires more data than the Pune, method used in Hong Kong (China) can capture the actual driving pattern in the area to a greater extent. Major Limitation of this method is that accuracy is intensively depend on the quality of the survey and the sample selected. If the sample is not selected carefully the values obtained for traffic related parameters will dramatically vary from the actual situation, hence will be less accurate than selecting route based on the experience of the researcher. To increase the representativeness of the survey the sample size has to be increased and thus cost rise is inevitable. But for a country where the traffic related data is readily available this method can be adopted easily.

Different method has been adopted to develop ARTHEMIS driving cycle for European region where study routes have being selected randomly from France, UK and Germany. Unlike in Hong Kong (China), the drivers were given freedom to follow the traffic flow. A large data set was collected [12] compared to all the driving cycles developed around the world so far to increase the representativeness of the sample. Main advantage of this method is that less effort has to be given to select routes. Therefore this nature of route selection is most suitable when developing driving cycles for whole country or a lager region where there is complex road network. Nevertheless, the limitation of this method is that to increase the representativeness of the data sample, sample size as to be increased and the data collection method need to be modified.

Route selection method used by Australia, for Melbourne, Perth and Sydney are much rational than many route selection methods used around the world. Many parameters have been considered for route selection such as land use, road type, driving condition and availability of public transportation [19]. For Sydney driving cycle routes were selected according to road classification and the traffic density in the areas reported with highest emissions. Then the driving patterns were measured in these regions and sample size for data collection was determined by conducting an OD survey [28]. For Perth driving cycle [29], six regions have been identified for the study. Thirteen routes have been selected based upon best available data which describes the traffic behavior and the actual road condition of the routes in Perth city such as Vehicle Kilometer Travelled (VKT) and traffic volumes. The attempt is to cover the important routes in Perth city and to consider high and low volume roads. But the routes selected for this method is not meant to represent the actual route which might take by a general motorist but to provide rational sampling framework to represent the driving pattern in selected six regions. In Melbourne cycle, ten routes within $8 \mathrm{~km}$ radius of the Melbourne central business city was selected which covers central business district, arterial and freeway conditions [30]. One of the best route selection method was used by Australian Composite Urban Emission Driving Cycle. Data is collected from Brisbane, Sydney, Melbourne, Adelaide and Perth metropolitan areas. Routes were selected according to the travel time available to 
provide a representative sample of routes and travel environments within the metropolitan area and are based on the arterial road network [31]. Since the method has considered many traffic related parameters to select routes, it has a higher representativeness than many methods discussed in this section. But this method cannot be used in many countries, especially in the developing countries where there are no readily available traffic data base.

\subsection{Data Collection}

For any type of research, data collection plays a vital role because the quality of the output is directly influenced by the reliability, representativeness, homogeneousness and consistency of the data which have collected. With respect to data collection literature states two basic methods, namely, chase car method and on board measurement method [32]. Data can be collected combining those two methods and it is known as hybrid method [7]. When selecting a vehicle for data collection it is wise to consider the engine capacity of the vehicle, because the engine capacity of the vehicle would change the driving pattern, hence it will either underestimate or overestimate the pollution and fuel consumption [33].

\subsubsection{Chase Car Method}

Literature clearly shows that chase car method is one of the popular methods of data collection for driving cycle construction all over the world. In this method an instrumented vehicle which can measure second by second speed data is used to chase a target vehicle in a predetermined route(s) within an area of concern [34]. Its drivers' responsibility to follow the target vehicle in the same way it travels to collect accurate data and this method is very difficult on roads where LOS is low. For LA92 and LA01, driving cycles developed in USA, the chase car method was adopted and the driving data was collected in greater metropolitan Los Angeles area [35]. In Pune; India, data was collected during peak hours in five major roads [25]. But collecting data only in peak hours may overestimate the traffic behavior of that area. An answer to this was given in Edinburg cycle [36] where data has collected at both peak and off peak hours proportionate to the traffic volumes.

One of the major advantages of the chase car method is that it requires lesser sources. Hence it is much more cost effective than using on-board measurement method. Due to cost consideration the chase car method was adopted by Manila driving cycle and collected approximately 6.4 hours of travel data [37]. But the sample size used to develop Manila driving cycle is limited compared to other sample sizes considered in other driving cycle developments. Major limitation of this method is that it is difficult to chase a car when driving behaviors are aggressive, where the chase car might be lost sometimes or chasing will lead to accidents.

\subsubsection{On Board Measurement Method}

In on board measurement method an instrument is installed in selected vehicles and let the vehicles travel along the traffic stream in selected routes. Then second by second speed data is recorded as they travel along the predetermined routes.

In on board measurement method major concern has to be given for the route selection. If random routes are adapted the size of the data sample should be increased as in ARTHEMIS cycle. In the ARTEMIS driving cycle the data was collected using on board measurement method using 77 vehicles. To increase the accuracy of the data sample 2200 hours of data was recorded, covering 2000 days (5.5 years). And vehicle usage and operating conditions were also reported [38], which was as same as in Australian Composite Urban Emissions Drive Cycle (CUEDC). In CUEDC sample size has increased to 431.4 hours to enhance the accuracy in considerable amounts [31]. This method is not easy to adopt by many countries since it requires more instruments as well as long period of monitoring, and hence costly.

One of the driving cycles developed for Hong Kong (China) in 1999, data acquisition was done using optical sensor pointing to the axle of the vehicle [11]. The rotation of the wheel is measured by the infrared photoelectric senor and pulse convertor. Then the speed data were collected in the micro-computer. To capture the critical scenario data was collected during peak period. But in another driving cycle developed for Hong Kong in 2007, data were collected using on board measurement method combined with chase car method using GPS loggers [19]. 29 hours of data were collected with the chase car method and 5.6 hours of data was collected using on board measurement method along nine selected representative routes during the morning peak hours in different routes, in which 44 were urban, four were sub-urban, and 26 were highway speed profiles. Also for the Bangkok driving cycle and driving cycles for motorcycles and light-duty vehicles in Vietnam, data was collected during 
peak hours. In Bangkok driving cycle data was collected using a real time logging system equipped on a selected vehicle traveling along the routes under actual traffic and the speed time data was collected during morning peak period to capture the driving condition which has the largest impact on exhaust emission and fuel consumption. The data collection of each route was carried out for two weeks [26]. When developing driving cycles for motorcycles and light-duty vehicles in Vietnam data was collected using on board measurement method and totally 60 trips 40 trips were representing motorcycle profiles and 20 trips were light duty vehicle profile [27]. Compared to ARTHEMIS and CUEDC driving cycles, required sample sizes for this method is less and if the routes are selected accurately the representativeness can be maintained properly while keeping the sample size minimum.

This method is suitable for the countries where the driving behavior is irregular and aggressive. Also for the countries which have traffic related databases to accurately select the routes. Unlike in chase car method, if the routes were not selected accurately the results can vary dramatically from the actual behavior of the region. Even though the method is costlier than chase car method, this method can be used economically while maintaining the data sample minimum if the routes are selected accurately using available traffic data base.

For the countries have mixed traffic behavior such as aggressive/congested and not congested roads (major congested highways and sub urban uncongested roads) the hybrid method can be used to optimize the cost of data collection while maintaining the representativeness of the data sample.

\subsection{Cycle Construction}

Method of cycle construction varies with the purpose of the driving cycle, estimation of emission inventories, estimation of fuel consumption or for traffic engineering purposes. Each cycle construction method has its unique features to represent its’ envisioned purpose.

As an example when developing driving cycles for emission and fuel estimation it is necessary to consider the cold start if the country has a cold or snowy weather conditions. Temperature is a key parameter to determine emission and fuel consumption and needs to be monitored consistently [39] [40]. In some cases, the hydrocarbons emit by average Euro-4 gasoline vehicle within its hot run during 300 to $2500 \mathrm{~km}$ is equal to single cold start [40]. Also if it is for the traffic engineering purposes the cycle should represent the actual point on the road for given speed or time. Hence a driving cycle developed for emission study purpose may not suit for traffic engineering purposes. The driving cycles developed in early stages in US, FTP72 and FTP75 the researchers have selected the whole trip which is best fit to the overall survey data. But sometimes these results are varied from the actual situation on the road. Improvements in driving cycle development leads to four major driving cycle construction methods, namely, micro-trip based cycle construction, segment based cycle construction, pattern classification cycle construction and Modal cycle construction [41].

\subsubsection{Micro-Trip Based Cycle Construction}

Micro trip based cycle construction can be defined as driving activity between adjacent stops, including leading idle period [41]. In this method the real data is divided in to micro trips and assigned in to different bins according to average speeds. The cycle is constructed using micro trips such that the target parameters are met (population parameters). For selecting micro trips for the cycle there are two main methods, namely, random selection and best incremental method [41]. In addition some driving cycles have used hybrid of those two methods as well.

But in micro trip based cycle construction the major limitation is that it is not possible to differentiate micro trips by various types of driving conditions such as roadway type or Level of Service (LOS). Therefore it is limited to develop cycles designed to represent a single type of trip or cycles designed to replicate region-wide driving conditions [41]. It is a well-known fact that more fuel is consumed by vehicles during "stop-go" conditions. Traffic signals and congestion generate most of the "stop-go" driving patterns and lead to higher fuel consumption [42]. Since micro trip based cycle construction covers each stop-go condition it will be a better approach for emission purpose and fuel estimation purpose.

LA92 was developed using micro trip based cycle construction method and quasi random method for micro trip selection [35]. The Bangkok driving cycle was also developed using micro trip based cycle construction and micro trips were selected randomly from the database [26]. In primary driving cycle developed for Colombo, Sri Lanka, the whole trip was first divided in to micro trips and then assigned in to bins considering their average speeds. From the bins micro trips were selected using best incremental method by considering 12 target para- 
meters. A tolerance limit of $\pm 5 \%$ was considered when choosing micro trips for the candidate cycles. Similar method was followed in Hong Kong (China) driving cycle, but the parameters considered were different. Even though the micro trip method is used in Manila driving cycle, selection of micro trips were done using statistical methods such as Fourier series, time series analysis and curve fitting techniques using polynomial functions [37].

Pune driving cycle used a speed acceleration matrix for each micro trip as well as for the population. Using the matrix target parameters such as time proportions and averages were generated for each micro trip as well as for the population. When selecting micro trips for the candidate cycle, calculated parameters of the micro trips were matched with the population target parameters and the tolerance limit was given as $5 \%$ for lower limit and $15 \%$ for upper limit [25]. This tolerance limit is better to develop driving cycle for emission purposes because it allow for $15 \%$ overestimation and only $5 \%$ under estimation. If the tolerance limit is decreased the representativeness will be increased. But then to fulfill the requirement, data sample should be increased hence the cost will be increased.

\subsubsection{Segment-Based Cycle Construction}

Segment based cycle construction is also similar to micro trip based cycle construction but there the roadway type or LOS is considered when selecting a trip "segment" instead of adjacent stops [41]. As a result the trip segments can represent the actual traffic condition as well as the physical characteristics of the road and LOS. This method is most suitable for a driving cycle for traffic engineering purposes but not for emission purpose [41]. In this method, since trip segments are divided according to traffic condition and physical characteristic of the road, it can be started and ended at any speed. Therefore when chaining the trip segments in to driving cycle it is necessary to match the speed and the acceleration between two consecutive connecting points (seconds in time) in micro-trips.

Segment based cycle construction has been used to developed Australian Composite Urban Emissions Drive Cycle (CUEDC). Researchers have identified the road categories as congested, residential/minor, and arterial and freeway/highway and each micro trip was assigned to one of the four road categories based on the average speed of the micro trip and the proportion of idle time in the micro trip. Then driving cycle was developed using target parameters similar to micro trip based cycle construction method [31]. But it is much easier to construct emission related cycles using micro trip based construction method or using an advanced method such as pattern classification or modal cycle construction method.

Segment based cycle construction found to be more appropriate for developing a driving cycle for expressways. The main underlying reason is that in an expressway there are no adjacent stops between origin and destination hence impossible to use Micro trip method. Therefore segment based method can be used for the regions or roads where there are less number of stop-go conditions or where the durations of the adjacent stops are presumably long.

\subsubsection{Cycle Construction with Pattern Classification}

In this method whole trip is divided into heterogeneous classes using statistical method and when dividing the trip "kinematic sequence" is considered. Succession probability is used for the estimation and considered likelihood of the event which could occur after selected activity. Driving cycles are constructed by random selection of kinematic sequence from segmented activity classes considering the probability and chronology of kinematic sequences [12] [41]. This method too has some limitations such as not directly related to emissions and it might not suite for an emission related driving cycle even though the classification of sequences is based on the chi-square distance of speed-acceleration joint distribution [41].

Pattern classification cycle construction method is highly statistics based construction method and many of European driving cycles have been developed using this method or at least partially using this method. ARTEMIS driving cycle was developed using this method and have classified kinetic sequence in to heterogeneous classes using 12 parameters. Succession probabilities were used to estimate and consider the likelihood that one class of activity precedes or follows a different activity class. Driving cycles are constructed by reconnecting kinematic sequences randomly selected from each of the activity classes in accordance with the probability and chronology of kinematic sequences [12].

One of the major drawbacks of this method is that when adopting this method at an early stage of driving cycle development it requires more information to divide collected data into kinematic sequences and to classify 
the routes, hence time consuming. However, this method has the ability to capture the traffic pattern on the road and could closely represent the actual behavior on a road, region or country.

\subsubsection{Modal Cycle Construction}

In modal cycle construction the real world driving patterns are divided in to acceleration, deceleration, cruising and idling components. To construct the driving cycles, Markov Chain theory is used assuming that likelihood of particular modal event depends only upon the pervious modal event [43]. There are four basic steps for constructing driving cycle using modal cycle construction method [41].

1) Using Maximum Likelihood clustering method collected data is divided into the snippets based on acceleration.

2) Snippets are assigned in to modal bins using Maximum Likelihood clustering method according to average, minimum, and maximum speeds and acceleration rates.

3) Define modes according to the acceleration rates and average speed, and then develop a transition matrix that contains the succession probabilities between different modes.

4) Using Markov chain snippets are chained in to one cycle considering transition probability matrix.

To develop LA01 cycle Maximum Likelihood estimation is used to partitioned route based on instantaneous speed into snippets of variable durations and intensities. Then the data collected are clustered it in to modal bins and a modal event bin, labeled by speed and acceleration. All the modal event bins combined define a set of states and form the state space of a Markov process. Target parameters such as average speed, maximum speed, maximum acceleration, and idle time percentage and road power, which is positively correlated with emission (road power is a function of travel speed and acceleration rate), along with speed acceleration frequency distribution (SAFD) of the candidate cycle is compared with the population and matches the end speed of the previous modal event with its starting speed which is chosen from the modal event bin. This selection process is repeated until the desired cycle length is achieved [43].

Same as in Pattern classification method this method represent the actual traffic condition because to chain the modal bins it uses probability of occurrence of each mode on the road. But the limitation of this method is that if the traffic behavior of the road or in the area is smooth then probability of occurrence matrix has some gaps or the duration of a modal event is much longer compared to the total length of the cycle. Hence this method cannot be used or if used it is difficult to get a candidate cycle which is closely match to the population parameters.

\subsection{Cycle Assessment}

Cycle assessment is one of the major steps in developing driving cycles, because the assessment criteria assures that the developed cycle truly represents the actual driving pattern on road in that particular area or region. Many countries have used many methods and parameters to assess their developed cycles. In common, many countries have used Performance Value (PV) to assess their cycles. For PV calculation it is necessary to calculate target parameters for population and the target parameters for candidate driving cycles. The percent deviation is calculated between target parameters of population and candidate driving cycles. Finally the summation of the absolute values of the deviations is taken after multiplying each absolute deviation by a weightage. This weightage is given according to the relevance of those parameters to the purpose of the driving cycle.

At early stages of driving cycle development, in FTP72 and FTP75, number of stops per distance, average speed, and maximum speed were used as assessment criterions. But driving cycles developed in recent past have used some common categories of parameters such as maximums and minimums, time proportions, averages, standard deviations, percentiles and some specific parameters for emission such as Root Mean Square Acceleration (RMS), Vehicle Power (P) and Positive acceleration Kinetic Energy (PKE) which could capture the actual situation in considered area or a road. Table 1 summarizes the commonly used major statistical categories for cycle assessment and appropriate traffic related target parameters under each category in the data set. Also this section further describes the parameters identified under each sub category, but not included the parameters which are not commonly used for assessment.

\subsubsection{Maximums and Minimums}

Hefei driving cycle in China has used maximum and minimum accelerations as one of the assessment criterion 
Table 1. Commonly used traffic related target parameters for cycle assessment.

\begin{tabular}{|c|c|}
\hline Sub category & Traffic related target parameter \\
\hline Maximum and minimums & Speed, acceleration, deceleration, acceleration rate. \\
\hline Percentages & Idle time, acceleration time, deceleration time, cruise time, creeping time. \\
\hline Means and averages & $\begin{array}{l}\text { Average speed, average running speed, average acceleration and average deceleration, mean length } \\
\text { of a driving period, average number of acceleration deceleration changes within one driving period. }\end{array}$ \\
\hline Standard deviations and percentiles & $\begin{array}{l}\text { Speed standard deviation and acceleration standard deviation, speed at the 95th percentile, } \\
\text { acceleration/deceleration rate at the } 95^{\text {th }} \text { percentile. }\end{array}$ \\
\hline Other emission related parameters & $\begin{array}{l}\text { Root mean square acceleration, Positive acceleration Kinetic Energy, Speed Acceleration } \\
\text { Probability Distribution (SAPD) or Speed Acceleration Frequency Distribution. }\end{array}$ \\
\hline
\end{tabular}

[44]. Whereas maximum speed and maximum deceleration was used in Chennai to assess their driving cycle developed for intra city busses [9]. The LA01 driving cycle has used maximum acceleration rate along with maximum and minimum speeds for assessment [35]. Further, Manila driving cycle has also used maximum and minimum speeds as one of the criteria to assess their cycle [37].

\subsubsection{Percentages}

LA01 and Manila driving cycles used percentage idle time and Bangkok driving cycle used percentages of number of micro trips. But commonly used percentages or proportions for assessment criteria are idle time proportion, acceleration time proportion, deceleration time proportion and cruise time proportion, which were used for driving cycles in Hefei (China), Hanoi; Vietnam, Pune; India, Hong Kong (China), and Bangkok. Moreover in driving cycle for intra city busses in Chennai percentage of time spent in creeping mode has also considered other than previously mentioned four parameters [9].

\subsubsection{Means and Averages}

Most frequently used averages are average speed, average running speed, average acceleration and average deceleration. Those parameters have been used to assess Hefei driving cycle; China (except average deceleration), Hanoi driving cycle; Vietnam, Bangkok driving cycle, Manila driving cycle; Philippine and driving cycle for intra city busses in Chennai; India.

Other than the above mention parameters mean length of a driving period, average number of acceleration/ deceleration changes within one driving period is also used for some driving cycle assessments such as in Hanoi driving cycle and average acceleration/deceleration rate in LA01 [35].

\subsubsection{Standard Deviations and Percentiles}

Hefei driving cycle has used standard deviations such as speed standard deviation and acceleration standard deviation [44] and LA01 has used percentiles such as speed at the $95^{\text {th }}$ percentile, and acceleration/deceleration rate at the $95^{\text {th }}$ percentile [35].

\subsubsection{Specific Parameters}

Not only statistical parameters but also some parameters derived from speed, time and acceleration/deceleration changes have used to assess candidate cycles such as average road power in Hefei and LA01 cycles, Root mean square acceleration (RMS) in Hanoi driving cycle [27] and Positive acceleration Kinetic Energy (PKE) in Hanoi, Bangkok and Hong Kong (China) driving cycles.

Most of the countries have used ten to thirteen parameters or less to assess driving cycles. But the Australian Composite Urban Driving Cycle (CUEDC) has used 46 parameters [31]. There are six parameters to represent the proportion of time spent in each mode such as proportion time idle, proportion time acceleration from idle, proportion time deceleration to idle, proportion time inter-acceleration, proportion time inter-deceleration, and proportion time cruise. Instead of using average values CUEDC has used the median values such as median idle time, median time acceleration from idle, median time deceleration to idle, median time inter-acceleration, median time inter-deceleration, median time cruise to avoid the effects of outliers in data sets and then the standard deviation has been calculated for each parameter to understand the variation from the population. CUEDC has 
used some parameters to represent the median magnitude of acceleration from idle, deceleration to idle, interacceleration, inter-deceleration, and cruise along with their standard deviation. Furthermore, median value of trip distance, total trip time, average trip speed, idles, trip PKE, max speed trip, trip non idle speed, max trip acceleration and min trip deceleration with their standard deviation with population are also considered for the assessment.

After assessing all the candidate driving cycles using above parameters a single driving cycle is selected, which gives a minimum PV, but if there are more than one candidate cycle with approximately equal PVs, then the SAPD/SAFD is used for further assessment.

\subsubsection{SAPD or SAFD}

Speed Acceleration Probability Distribution (SAPD) or Speed Acceleration Frequency Distribution (SAFD) is one of the common ways of assessing candidate driving cycles. In this assessment acceleration vs speeds vs probability is plotted in a 3D graph. In order to plot the graph, speeds are segmented in $5 \mathrm{~km} / \mathrm{h}$ and acceleration in $1 \mathrm{~km} / \mathrm{h} / \mathrm{s}$ [29].

Some driving cycles have used SAPD or SAFD for the selection of driving pattern to construct their driving cycle. In LA92 and LA01 SAFD was used as target parameter. By looking at the population SAFD the micro trip was selected for the cycle construction [35].

But in most cases such as in Hefei (China), Hanoi; Vietnam, Sydney, Istanbul, driving cycle for intra city busses in Chennai; India, Manila; Philippine, Hong Kong (China) and ARTEMIS driving cycles SAPD/SAFD is used to assess the candidate driving cycle with the population statistics. First SAPD/SAFD is created to whole population and then construct SAPD/SAFD for each candidate cycles. The most representative driving cycle was determined by the smallest sum square difference (SSD) between the SAPD/SAFD of the candidate cycle and the overall SAPD/SAFD.

\section{Conclusions}

When selecting a method of developing a driving cycle for a country or a region there are two major non-technical constraints to consider, namely, cost and time. Therefore when developing a basic driving cycle, a suitable method(s) should be selected from each step optimizing those constraints. However, it should be noted that each step has its own advantages and disadvantages inherited as discussed in this paper. Therefore all facts must be considered carefully when selecting different approaches for each step based on the objective of driving cycle development. Route selected is highly correlated with time taken for data collection; because if a complex route network is selected the time taken will be increased to collect data since many road sections are present. In order to select routes the information required will be higher if there is no readily available traffic information the required data have to be collected which increases the cost. On the other hand, a simple method could be adopted; then the representativeness of the collected data will be inferior. For a developing country where the driving cycle development is at the preliminary stage it is advisable to use a simple method where data requirement is less hence the cost for data collection is less. Experience of the researcher about the traffic behavior of the region could be a predominant factor in such instances in order to select representative routs as did in many countries. Furthermore if the simple traffic related information such as origin destination data or traffic counts is available, it is advisable to merge such information with the experience of the researcher to increase the reliability of the route selection process. If the accuracy is highly required, surveys have to be done to collect traffic related data such as origin destination of the vehicles, traffic counts, and vehicle kilometer travel and vehicle proportions. Furthermore details of the physical properties of the road such as surface condition and type, number of lanes, number of signalized and non-signalized intersection and availability of center median have to be collected.

As for the literature the economical way of data collection is chase car method where it requires limited resources. Therefore this method is suitable for countries with limited budgets. Also for the chase car method, route selection is not vital since the car follows the actual road user. Nevertheless the data sample might be bias hence the cycle developed will be less representative to the actual situation but that problem can be overcome by increasing the sample size of the data set, then again the cost will be high. Therefore it is better to prioritize the precision/representativeness of the driving cycle or the budget available and then optimize the process. Limitation of the method is that for the areas where the driving pattern is aggressive this method cannot be used. For 
the countries which have required information to accurately select representative routes and the budget is flexible an onboard method can be used. Representativeness of this method can be further increased by increasing the sample size. Furthermore collecting data proportionate to the traffic volume and collecting data in different time periods of the day proportionate to the corresponding volumes in off peaks and peaks could also improve the representation. But in the initial stage if the critical emission is required data can be collected in major roads during peak hours.

Cycle construction method has to be selected carefully because the selected method would determine the sample size required for data collection. Sample size has a significant effect on the total cost of the driving cycle development. For a country where the driving cycle development at its preliminary stage it is advisable to use micro trip cycle construction for emission purpose if the selected area has many stop go conditions because it is simple and will provide a representative cycle for emission purpose. If there are less stop go conditions and the speed are not gradually varying (if the driving behavior is aggressive but no stop go conditions) the best method will be modal cycle construction. But in modal cycle construction the data sample size needs to be increased to capture the states of driving defined in the method to fill the transition probability matrix; hence the data collection will be costly. Therefore it is most suitable for the countries which require more representativeness of their driving cycle. Finally segment based cycle construction method can be used for road segments like expressways where there is smooth variation of speeds and less number of stop go conditions. Furthermore this method can be used for traffic engineering purposes as well.

Cycle evaluation parameters should be selected carefully because due to number of parameters used the data required (sample size) are increased. When driving cycle development is at preliminary stage basic parameters that are commonly used in many countries can be used for cycle evaluation. The common parameters are, namely, average speed, average running speed, average acceleration, average deceleration, percentage of idling, percentage of acceleration, percentage of deceleration, percentage of cruising, route mean square acceleration (RMS) and positive kinetic energy (PKE). Then the cycle can be compared using performance value (PV) and the cycle with minimum PV value can be selected as the representative driving cycle. Furthermore if more representativeness is required smallest sum square difference (SSD) between the SAPD/SAFD of the candidate cycle and the population data set should be considered in the candidate cycles where the PV is approximately equal. But if the number of parameters is less for the evaluation, the representativeness of the candidate cycle to the actual situation will differ. It is recommended to use PKE and RMS for the emission related driving cycle and use PV and SAPD or SAFD for the evaluation.

If the required accuracy is high, more parameters can be used; but then to match all the parameters the sample size needed to be collect is also high; hence the cost will be increased.

\section{Acknowledgements}

Authors are thankful to Dr. Thusitha Sugathapala for the guidance and motivation given and further thank goes to Sustainable Energy Authority for the resources provided for successful completion of this research.

\section{References}

[1] Zhang, X., Zhao, D.-J. and Shen, J.-M. (2012) A Synthesis of Methodologies and Practices for Developing Driving Cycles. Energy Procedia, 16, 1863-1873.

[2] Pandian, S., Gokhale, S. and Goshal, A.K. (2009) Evaluating Effects of Traffic and Vehicle Characteristics on Vehicular Emissions near Traffic Intersections. Transportation Research Part D, 14, 180-196. http://dx.doi.org/10.1016/j.trd.2008.12.001

[3] Joumard, R., Philippe, F. and Vidon, R. (1999) Reliability of the Current Models of Instantaneous Pollutant Emissions. The Science of the Total Environment, 235, 133-142. http://dx.doi.org/10.1016/S0048-9697(99)00202-8

[4] Zamboni, G., Carraro, C. and Capobianco, M. (2011) On-Road Instantaneous Speed Measurements on Powered TwoWheelers for Exhaust Emissions and Fuel Consumption Evaluation. Energy, 36, 1039-1047. http://dx.doi.org/10.1016/j.energy.2010.12.004

[5] Ericsson, E. (2001) Independent Driving Pattern Factors and Their Influences on Fuel-Use and Exhaust Emission Factors. Transport Research Part D, 6, 325-345. http://dx.doi.org/10.1016/S1361-9209(01)00003-7

[6] Freij, K.B. and Ericsson, E. (2005) Influence of Street Characteristics, Driver Category and Car Performance on Urban Driving Patterns. Transportation Research Part D, 10, 213-229. http://dx.doi.org/10.1016/j.trd.2005.01.001 
[7] Tong, H.Y. and Hung, W.T. (2010) A Framework for Developing Driving Cycles with on Road Driving Data. Transport Review, 30, 589-615. http://dx.doi.org/10.1080/01441640903286134

[8] Barlows, T.J., Latham, S., McCrane, I.S. and Boutler, P.G. (2009) A Reference Book of Driving Cycles to Use in Measurement of Road Vehicle Emission. 3rd Edition, TRL.

[9] Nesamani, K.S. and Subramanian, K.P. (2011) Development of a Driving Cycle for Intra-City Buses in Chennai, India. Atmospheric Environment, 45, 5469-5476. http://dx.doi.org/10.1016/j.atmosenv.2011.06.067

[10] Jeon, C.H., Han, D.S., Choi, N.W., Cho, S.L., Yang, J.S., Kim, K.S. and Yoo, W.S. (2012) Characterization of Driving Patterns and Development of a Driving Cycle in a Military Area. Transportation Research Part D, 17, 519-524. http://dx.doi.org/10.1016/j.trd.2012.06.004

[11] Tong, H.Y., Hung, W.T. and Cheung, C.S. (1999) Development of a Driving Cycle for Hong Kong. Atmospheric Environment, 33, 2323-2335. http://dx.doi.org/10.1016/S1352-2310(99)00074-6

[12] Andre, M. (2004) Real-World Driving Cycles for Measuring Cars Pollutant Emissions-Part A: The ARTEMIS European Driving Cycles. INRETS-LTE, Cedex.

[13] Tamsanya, N. and Chungpaibulpatana, S. (2009) Influence of Driving Cycles on Exhaust Emissions and Fuel Consumption of Gasoline Passenger Car in Bangkok. Journal of Environmental Sciences, 21, 604-611. http://dx.doi.org/10.1016/S1001-0742(08)62314-1

[14] Gamalath, I.M., Galgamuwa, U.N., Fernando, C.M., Perera, L. and Bandara, J.M.S.J. (2012) Methodology to Develop a Driving Cycle for a Given Mode and Traffic Corridor; Case Study for Galle Road, Colombo, Sri Lanka. Proceedings of the Civil Engineering Research for Industry Symposium, Moratuwa, 45-50. http://dl.lib.mrt.ac.lk/handle/123/9920

[15] Andre, M. and Mario, R. (2009) Analysis and Modelling of the Pollutant Emissions from European Cars regarding the Driving Characteristics and Test Cycles. Atmospheric Environment, 43, 986-995. http://dx.doi.org/10.1016/j.atmosenv.2008.03.013

[16] Hung, W.T., Tong, H.Y. and Cheung, C.S. (2005) A Modal Approach to Vehicular Emissions and Fuel Consumption Model Development. Air and Waste Management, 55, 1431-1440. http://dx.doi.org/10.1080/10473289.2005.10464747

[17] Kumar, R., Durai, B.K., Saleh, W. and Boswell, C. (2011) Comparison and Evaluation of Emissions for Different Driving Cycles of Motorcycles: A Note. Transportation Research Part D, 16, 61-64. http://dx.doi.org/10.1016/j.trd.2010.08.006

[18] Vehicle Chassis Dynamometer Driving Schedules, EPA. http://www.epa.gov/nvfel/testing/dynamometer.htm\#vehcyclesretrieved14/7/201

[19] Hung, W.T., Tong, H.Y., Lee, C.P., Ha, K. and Pao, L.Y. (2007) Development of a Practical Driving Cycle Construction Methodology: A Case Study in Hong Kong. Transportation Research Part D, 12, 115-128. http://dx.doi.org/10.1016/j.trd.2007.01.002

[20] Barrios, C.C., Dominguez-Saez, A., Rubio, J.R. and Pujadas, M. (2012) Factors Influencing the Number Distribution and Size of the Particles Emitted from a Modern Diesel Vehicle in Real Urban Traffic. Atmospheric Environment, 56, 16-25. http://dx.doi.org/10.1016/j.atmosenv.2012.03.078

[21] Bishop, J.D., Axon, C.J. and McCulloch, M.D. (2012) A Robust, Data-Driven Methodology for Real-World Driving Cycle Development. Transportation Research Part D, 17, 389-397. http://dx.doi.org/10.1016/j.trd.2012.03.003

[22] Ericsson, E. (2000) Variability in Urban Driving Patterns. Transportation Research Part D, 5, 337-354. http://dx.doi.org/10.1016/S1361-9209(00)00003-1

[23] China, S. and James, D. (2012) Influence of Pavement Macrotexture on PM10 Emissions from Paved Roads: A Controlled Study. Atmospheric Environment, 63, 313-326. http://dx.doi.org/10.1016/j.atmosenv.2012.09.018

[24] Nagpure, A.S., Gurjar, B.R. and Kumar, P. (2011) Impact of Altitude on Emission Rates of Ozone Precursors from Gasoline Driven Light-Duty Commercial Vehicles. Atmospheric Environment, 45, 1413-1417. http://dx.doi.org/10.1016/j.atmosenv.2010.12.026

[25] Kamble, S.H., Mathew, T.V. and Sharma, G.K. (2009) Development of Real-World Driving Cycle: Case Study of Pune, India. Transportation Research Part D, 14, 132-140. http://dx.doi.org/10.1016/j.trd.2008.11.008

[26] Tamsanya, N., Chungpaibulpattana, S. and Atthajariyakul, S. (2006) Development of Automobile Bangkok Driving Cycle for Emissions and Fuel Consumption Assessment. Proceedings of the 2nd Joint International Conference on Sustainable Energy and Environment, Bangkok, 21-23 November 2006.

[27] Tong, H.Y., Tung, H.D., Hung, W.T. and Nguyen, H.V. (2011) Development of Driving Cycles for Motorcycles and Light-Duty Vehicles in Vietnam. Atmospheric Environment, 45, 5191-5199. http://dx.doi.org/10.1016/j.atmosenv.2011.06.023

[28] Kent, J.H., Allen, G.H. and Rule, G. (1978) A Driving Cycle for Sydney. Transportation Research, 12, 147-152. http://dx.doi.org/10.1016/0041-1647(78)90117-X 
[29] Lyons, T.J., Pitts, R.O., Blockley, J.A., Kenworthy, J.R. and Newman, P.W. (1990) Mortor Vehicle Emission Inventory for the Perth Airshed. Journal of the Royal Society of Western Australia, 72, 67-74.

[30] Kenworthy, J.R. (1986) Driving Cycle, Urban form and Transportation Energy. PhD Thesis, Environmental Science, Murdoch University, Perth.

[31] Zito, R. and Primerano, F. (2005) Drive Cycle Development Methodology and Results. Transport System Centre, University of South Australia, Adelaide.

[32] Tong, H.Y., Hung, W.T. and Cheung, C.S. (2000) On-Road Motor Vehicle Emissions and Fuel Consumption in Urban Driving Conditions. Air and Waste Management Association, 50, 543-554. http://dx.doi.org/10.1080/10473289.2000.10464041

[33] Andre, M., Joumard, R., Vidon, R., Tassel, P. and Perret, P. (2006) Real-World European Driving Cycles, for Measuring Pollutant. Atmospheric Environment, 40, 5944-5953. http://dx.doi.org/10.1016/j.atmosenv.2005.12.057

[34] Niemeier, D.A., Limanond, T. and Morey, J.E. (1999) Data Collection for Driving Cycle Development: Evaluation of Data Collection Protocols. Department of Civil and Environmental Engineering, Institute of Transportation Studies, University of California, Davis.

[35] Lin, J. and Niemeier, D.A. (2002) An Exploratory Analysis Comparing a Stochastic Driving Cycle to California's Regulatory Cycle. Atmospheric Environment, 36, 5759-5770. http://dx.doi.org/10.1016/S1352-2310(02)00695-7

[36] Booth, A.E., Muneer, T., Kieby, H., Kubie, J. and Hunter, J. (2001) The Measurement of Vehicular Driving Cycle with the City of Edinburgh. Transport Research Part D, 6, 209-220. http://dx.doi.org/10.1016/S1361-9209(00)00024-9

[37] Sigua, R.G. (1997) Development of Driving Cycle for Metro Manila. Journal of the Eastern Asia Society for Transportation Studies, 2-6.

[38] Andre, M. (2004) The ARTEMIS European Driving Cycles for Measuring Car Pollutant Emissions. Science of Total Environment, 334-335, 73-84. http://dx.doi.org/10.1016/j.scitotenv.2004.04.070

[39] Demuynck, J., Bosteels, D., De Paepe, M., Favre, C., May, J. and Verhelst, S. (2012) Recommendations for the New WLTP Cycle Based on an Analysis of Vehicle Emission Measurements on NEDC and CADC. Energy Policy, 49, 234242. http://dx.doi.org/10.1016/j.enpol.2012.05.081

[40] Weilenmann, M.F., Soltic, P. and Hausberger, S. (2013) The Cold Start Emissions of Light-Duty-Vehicle Fleets: A Simplified Physics-Based Model for the Estimation of $\mathrm{CO}_{2}$ and Pollutants. Science of Total Environment, 444, 161-176. http://dx.doi.org/10.1016/j.scitotenv.2012.11.024

[41] Dia, Z., Eisinger, D. and Niemeier, D. (2008) Driving Cycles: A New Cycle-Building Method That Better Represents Real-World Emissions. University of California, Davis.

[42] Chen, C.H., Huang, C., Jing, Q.G., Wang, H.K., Pan, H.S., Li, L., Zhao, J., Dai, Y., Huang, H.Y., Schipper, L. and Streets, D.G. (2007) On-Road Emission Characteristics of Heavy-Duty Diesel Vehicles in Shanghai. Atmospheric Environment, 41, 5334-5344. http://dx.doi.org/10.1016/j.atmosenv.2007.02.037

[43] Lin, J. and Niemeier, D.A. (2003) Regional Driving Characteristics, Regional Driving Cycles. Transportation Research Part D, 8, 361-381. http://dx.doi.org/10.1016/S1361-9209(03)00022-1

[44] Shi, Q., Zheng, Y., Wang, R.S. and Li, Y.W. (2011) The Study of a New Method of Driving Cycles Construction. Procedia Engineering, 16, 79-87. http://dx.doi.org/10.1016/j.proeng.2011.08.1055 